\title{
An Architecture Description Language for Dynamic Service-Oriented Product Lines
}

\author{
Seza Adjoyan \\ UMR CNRS 5506 / LIRMM \\ University of Montpellier \\ Montpellier, FRANCE \\ adjoyan@lirmm.fr
}

\author{
Abdelhak Seriai \\ UMR CNRS 5506 / LIRMM \\ University of Montpellier \\ Montpellier, FRANCE \\ seriai@lirmm.fr
}

\begin{abstract}
Reconciling Software Product Lines (SPL) and Service Oriented Architecture (SOA) allows modeling and implementing systems that systematically adapt their behavior in respond to surrounding context changes. Both approaches are complementary with regard to the variability and the dynamicity properties. Architecture Description Language (ADL), on the other hand, is recognized as an important element in the description and analysis of software properties. Different ADLs have been proposed in SOA or in SPL domains. Nevertheless, none of these ADLs allows describing variability and dynamicity features together in the context of service-oriented dynamic product lines. In this sense, our work attempts to describe the changing architecture of Dynamic Service-Oriented Product Lines (DSOPL). We propose an ADL that allows describing three types of information: architecture's structural elements, variability elements and system's configuration. Furthermore, we introduce context elements on which service reconfiguration is based.
\end{abstract}

Keywords-Architecture Description Language (ADL); ServiceOriented Architecture (SOA); Software Product Lines (SPL); dynamicity; variability; software architecture; Dynamic ServiceOriented Product Lines (DSOPL)

\section{INTRODUCTION}

Software Product Lines (SPL) and Service Oriented Architecture (SOA) have a common goal from a software development point of view; increase the reusability of existing assets rather than rebuilding new systems from scratch. SPL, on the one hand, allows the development of a family of products that share some common set of core assets [1], [2], [3]. Variability has always been a first concern in SPL studies [16]. According to [4], variability is the ability of a software artifact to quickly change and adapt for a specific context in a preplanned manner. SOA, on the other hand, is a special kind of software architecture, where the main architectural elements are coarse grained and loosely coupled services that are dynamically composable and inter-operable [5]. Being able to modify the architecture of a running system at such a high level of abstraction renders the system highly extensible, customizable and powerful [6].

Variability and dynamicity are core properties to develop complex adaptable software systems such as telecommunication, pervasive, crisis management, surveillance and security systems. In such systems, due to environment changes, a dynamic re-configuration should be carried out without having to re-deploy the whole system.
Combining SOA and SPL constitutes the answer to this need [7]. SOA offers, through its encapsulation property and its explicit interfaces, a solution for achieving dynamic product lines. SPL offers, via variability modeling, analysis and design of changing points in service-oriented architectures.

Architecture Description Language (ADL) is a formalism that allows the specification of system's conceptual architecture [8]. It enables architects to describe and validate systems against stakeholders' requirements from one side, and ease the development and implementation process of complex systems, from another side. It often has a graphical representation or plain text syntax. Conventional ADLs support only static architecture description [6]. Some ADLs provide special formalism for SOA to describe service dynamicity or for SPL to describe variability. Unfortunately no ADL supports the crosscutting SOA and SPL concepts.

To overcome this limitation, we propose an XML-based ADL that allows describing the architecture of a Dynamic Service-Oriented Product Line (DSOPL). It describes the four following elements: (i) the structural elements of a family of software products (i.e. services and connections), (ii) an architectural variability model (i.e. variability points and alternatives), (iii) context information, in addition to (iv) an architectural configuration model (i.e. reconfiguration rules based on context and variability). We choose to use XML as a description language to facilitate understandability and analysis of the described architecture. In addition, XML-based description facilitates tool-support design and interoperability.

The remainder of this paper is organized as follows: In section 2, we discuss related works regarding variability and dynamicity properties. In section 3, we characterize our proposed DSOPL-ADL's elements and demonstrate their utility through a running example. Finally, in section 4, we summarize our contribution and provide directions for future research.

\section{RELATED WORK}

\section{A. ADLs specifying dynamic properties}

A software architecture can be classified in terms of its capability of evolution into two categories: static or dynamic. A static architecture reflects the static structure of software and is completely specified at design time [6], whereas in dynamic architecture, system may evolve after its compilation [1]. In this type of architecture, in addition to specifying the 
system in terms of components, connectors and configurations, it should also specify how these components and connectors are evolved or reconfigured at runtime. This evolution of architecture at runtime may happen under several forms: adding/ removing composing elements, reconfiguring architecture (modifying connections), or upgrading existing composing elements (substitution of composing elements).

ADLs are used to describe the properties of static or dynamic software architecture. Among existing ADLs in the literature, only few of them support dynamic reconfiguration such as C2 [9], Darwin [10], $\pi$-ADL [11], Rapide [12], ACME/Plastik [13] and Dynamic Wright [14]. In order to describe a specific configuration in [13], the expression "on \{condition\} do \{operations\}" is used to toggle between different choices at runtime. To replace an instance of component at runtime, detach and attachments statements are used in operations part to respectively unlink and link components. In $\pi$-ADL [11], the architecture is considered dynamic since third party services can be discovered and bound to service broker at runtime.

\section{B. ADLs specifying variability properties}

Dynamic Software Product Line (DSPL) extends conventional SPL perspective by delaying the binding time of product's composing elements (i.e. features) to runtime. It produces autonomous and reconfigurable products that are able to reconfigure themselves to select a valid configuration during runtime [15]. Even though there is no concrete agreement of what aspects a dynamic SPL should exactly treat, most approaches agree that the main characteristic of any dynamic SPL framework is the runtime variability, which provides the following common activities at runtime: managing the dynamic selection of variants, autonomous activation/ deactivation of composing elements, substitution of composing elements and dependency and constraint checking of changed elements [22].

Few existing approaches were concerned about representing an architecture that encompasses variability [16]. $x A D L$ [17] is an ADL for modeling runtime and design-time architectural elements of software systems. It is defined as a set of XML schemas. XADL 2.0 integrates product lines concepts in the form of three schemas: versions, options, and variants schemas. Concerning the integration of product lines concepts within xADL; this approach suffers from the limitation of expressing constraints (i.e. requires, excludes) between elements of different variation points. Koala [18] defines "switches" in order to dynamically bind the selected component. The main limitation in Koala is its static nature; any deployed configuration cannot be changed at runtime and will require application recompilation, thus it is not suitable for dynamic architectures.

Otherwise, approaches that describe variability in product lines architecture are not based on the service-oriented style. Approaches such as [19], [20] describe system's architecture in terms of services whether in a dynamic or static ADL. Nevertheless, these ADLs are not able to describe variants.

\section{DYNAMIC SERVICE ORIENTED PRODUCT LINE ADL}

\section{A. Illustrative example}

We will use throughout the paper an illustrative example to exemplify concepts related to our proposed approach. This example is about a simplified online sales scenario between four actors; customer, retailer, warehouse and shipment services, as modeled in Fig. 1. The customer accesses retailer's website, browses the catalog, selects some items and commands an order. The retailer fulfills customer's order request and inquires the warehouse to prepare all items of the order. Once the order is prepared, the shipping service handles the delivery of items to the customer.

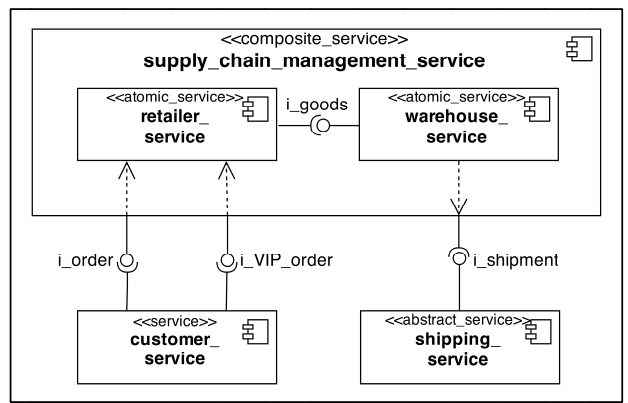

Figure 1. Illustrative example: online sales scenario architecture

\section{B. The DSOPL-ADL structure}

In order to describe the runtime variability of a Dynamic Service-Oriented Product Line (DSOPL) system at architecture level, we propose an XML-based ADL. This ADL is structured in four sections, as summarized in the schema of Fig. 2:

1. Structural element description: defines all types of the abstract structural entities of the system (services, interfaces, operations).

2. Variability description: here, variation points are defined and also all alternative services of each variation point with the constraints related to each alternative.

3. Context description: variability and configuration descriptions are based on information about context. Thus, information about context elements is described in a specific section of the ADL.

4. Configuration description: here, the rules used to create concrete services and connections are specified to describe how to configure (generate) concrete architectures based on structural, variability and context elements.

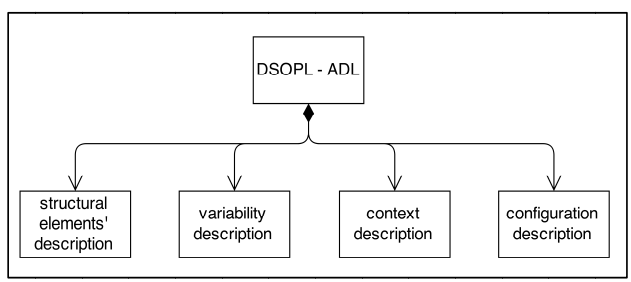

Figure 2. Modular DSOPL-ADL 
Our approach implicitly separates the four aforementioned architectural concerns from each other. The modular description of architecture in four sections, each of them specifying one type of architectural description has the following advantages:

1) It facilitates the modification and re-utilization of each of the four sections of ADL.

2) It allows the description and analysis of the architecture by separating the four concerns (structure, variability, context and configuration).

3) It allows controlling the traceability links of each type of information among several abstraction levels. For example, the variability described in feature model at requirement level is translated at architecture level through its variability section.

\section{Structural elements description}

A service is an encapsulated and self-contained unit. It interacts with other services through interfaces. The system itself is a composite service and is hierarchically decomposed into finer-grained services. Leaf services are called atomic services. All other services in the hierarchical tree are considered composite. A composite service doesn't execute or implement any functionality by itself, but it delegates this task to one of its composing services. Each composite service is described as sub-architecture.

Each service has a number of provided interfaces and may require a number of required interfaces. Interfaces define a collection of methods or operations that are supported by the service. Since services are developed independently from their future exploiting systems, they should have solid and welldefined interfaces that describe their functionalities and operations. Interfaces are two types, either provided interface or required interface. Provided interface of a service is an interface that the service realizes, whereas required interface is an interface that the service needs in order to operate. Services communicate to each other through provides/ consumes relationship via their provided/ required interfaces. An interface has a set of operations.

The structural description of a service reflects this service meta-model. A service is described based on the following architectural attributes, as shown in Fig. 3: (1) Every service has a name specified by service_name attribute. (2) It has a

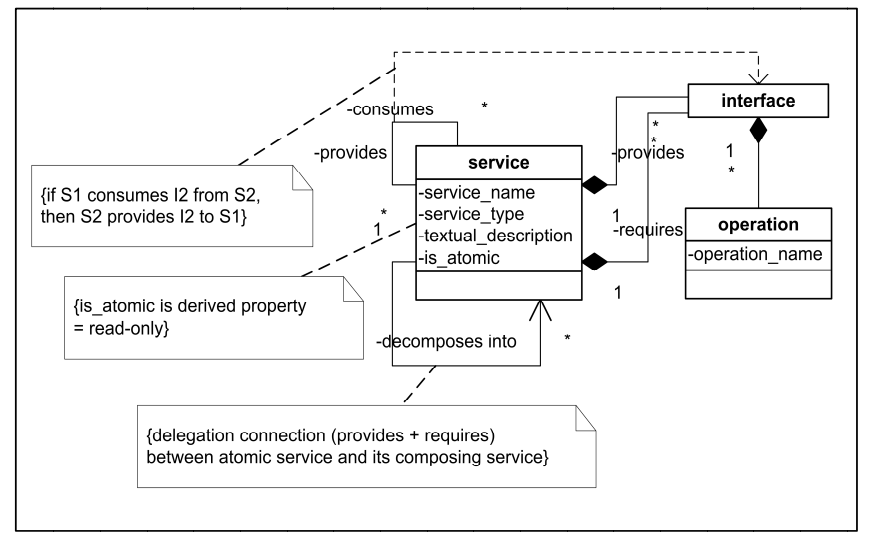

Figure 3. Structural description meta-model of DSOPL-ADL textual_description that explains in plain text the main functionalities of the service, its inputs and expected outputs. (3) is_atomic has a Boolean value to indicate whether the service is atomic or composite. Fig. 4 shows the structural section description of the architecture related to our illustrative example.

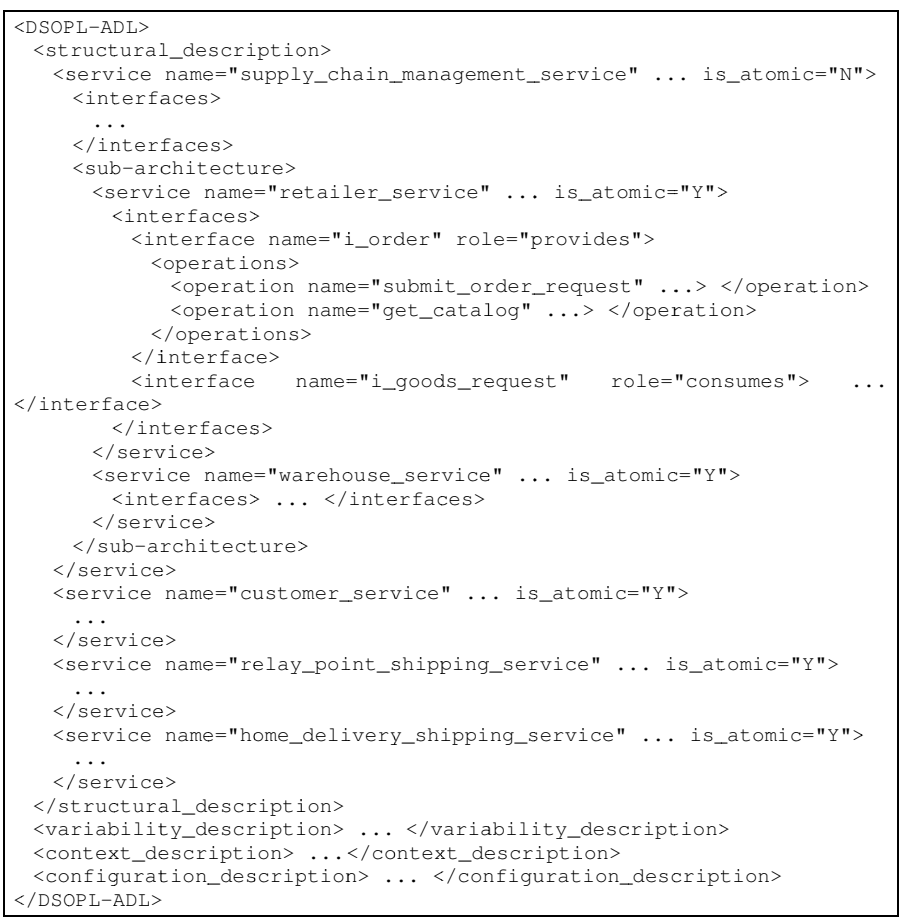

Figure 4. Structural description of sales scenario

\section{Variability description}

Variability in SPL architecture refers to the ability of making changes to system's architecture. We distinct three types of variability:

\section{1) Service variability}

It represents binding an alternative service that satisfies pre-conditioned constraints on runtime. Back to our sales example, there are two alternatives of shipment; either a relay point shipment or home delivery shipment, as shown in Fig. 5. The decision of which alternative to choose is taken automatically at runtime depending on customer's selection in addition to other environmental conditions such as the existence of a relay point service in customer's city, as depicted in Fig. 9.

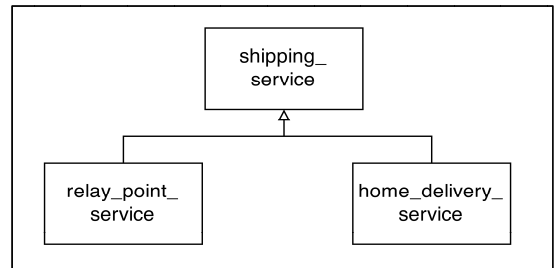

Figure 5. Example of service variability in sales scenario

2) Variability of connection

It may exist several alternative connections between services. The selection of the appropriate connection is done 
automatically at runtime according to constraints' satisfaction. For example, the customer service in Fig. 6 can access the retailer service and thus command an order via two different connections; either a connection for a regular customer or a connection for a VIP customer which normally has some extra privileges. The variation_point "customer_variation_point" in Fig. 9 is an example of variability of connection.

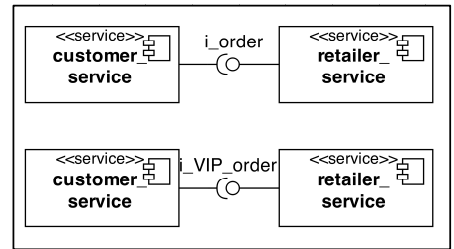

Figure 6. Example of connection variability in sales scenario

\section{3) Variability of composition}

This type of variability concerns replacing not only a service or a connection, but replacing a set of interconnected services by another set of interconnected services within a composite architecture. Fig. 7 illustrates another alternative composition of supply_chain_management_service than the one in Fig. 1. Here, in addition to the roles of retailer and warehouse services, the manufacturer service realizes requested items and returns them to the warehouse service.

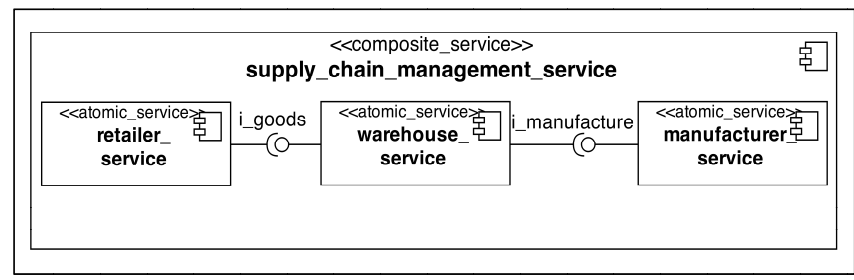

Figure 7. Example of composition variability in sales scenario

The meta-model of variability description is given in Fig. 8. We specify in this section the different variation points that exist in the system at architectural level. A variation point specifies the part of the architecture that can be variable. Each variation point has the following attributes: (1) variation_name indicating its unique name, (2) variation_type that specifies the type of this variation. Possible values of variation_type are either service, connection or composition. (3) variation_time specifies whether this variation may occur at compile-time (i.e. before runtime) or at runtime. Contrary to traditional SPL approaches where variability is clearly and completely specified at design time [21], variation_time attribute is important in SOA systems, where selection of an alternative during runtime is totally possible. However, some variation points could be specified at compile-time. This reduces the overhead of loading the entire configuration at runtime. Each variation point has several alternatives, which are possible elements to fill the selected variation point. Each alternative has a unique name alternative_name and an order of priority. This attribute helps the system automatically determine which architectural element is chosen in case there is more than one valid configuration at a given time. The alternative with the highest priority priority=" 1 " is the

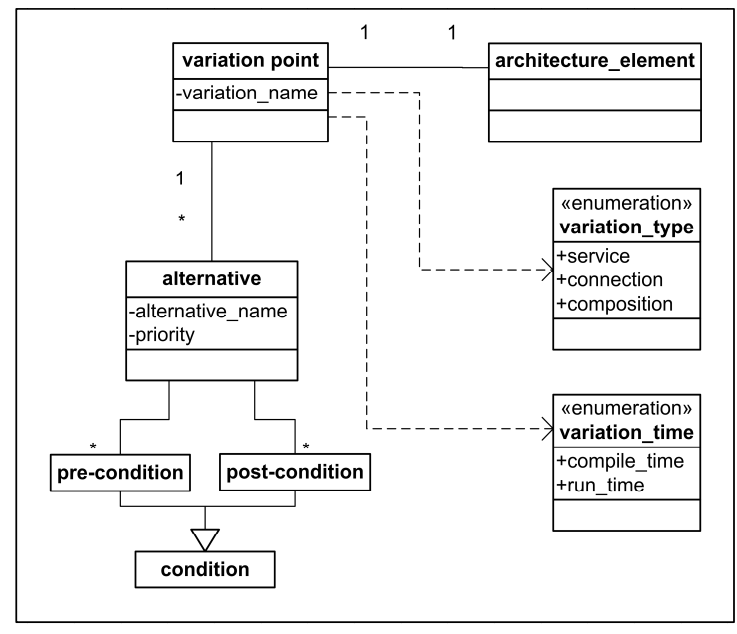

Figure 8. Variability description meta-model of DSOPL-ADL

preferred one in a variation point. Each alternative has a set of constraints, in forms of pre-conditions and post-conditions, to operate properly. Pre-conditions specify a group of conditions that should be satisfied before executing the selected alternative (i.e. alternative can be selected, only if all constraints of pre-conditions are satisfied). Post-condition represents desirable outcomes when process is completed successfully. Pre and Post-conditions are the equivalent of crosscutting "requires", "excludes" and "and" constraints in Feature Model FM in SPL paradigm. For example, the precondition that states that in order to choose the alternative "relay_point_delivery_alternative", the service "relaying_point_service_in_city" should be available (see Fig. 9), this statement is equivalent in FM to a "requires" constraint from "relay_point_delivery" feature to "relaying_point_in_city" feature. On the contrary, condition="unavailable" is equivalent to "excludes" constraint in FM.

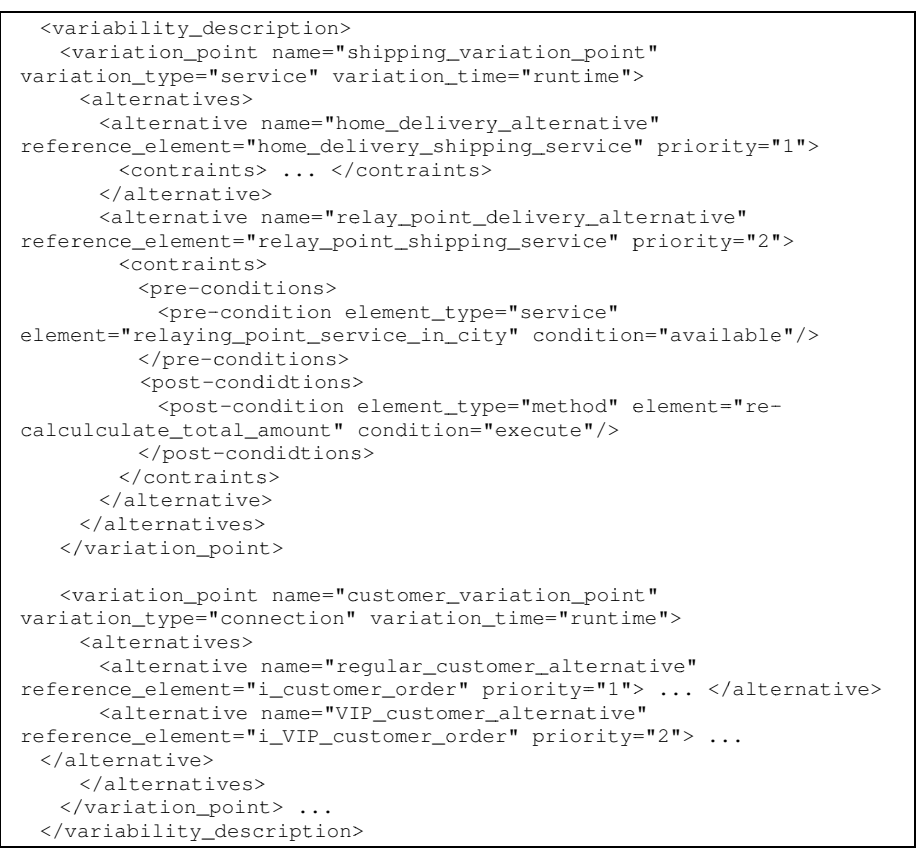

Figure 9. Variability description of sales scenario 


\section{E. Context description}

Architecture reconfiguration is based on context changes. The context consists of any element that influences the behavior and/or the structure of the architecture. It can be related either to system's environment (e.g. escalator state in the case of crisis management software), evaluated quality of service (e.g. time to response to a query), hardware architecture changes (e.g. server failure), etc. Thus, context element needs to be described in a dynamic ADL. We include these context elements as part of the architecture description to allow context-aware configurations (i.e. autonomous run-time adaptation according to context changes). A context element could capture raw data from a single information source such as a GPS locator that locates customer's current location to search for a nearby relay point for the shipping service in our sales example. In this case, context element is considered as a primitive_context. In some other cases, a single information source could not be sufficient to take decisions; in that case, different atomic information sources' values are collected, combined and analyzed in order to give sufficient and more accurate information about the context value. We call this context as composite_context. We can consider the weather forecast example, where the weather is considered hot when both temperature and humidity sensors exceed a certain threshold.

A simplified meta-model of context is illustrated in Fig. 10. Any context element has a unique name and a context_type to indicate to which family of contexts it belongs (e.g. contexts related to environment, user preferences, etc.). Context element also has values_type that indicates the type of its values, either primitive types such as integer, double, etc. or user-defined types. In Fig. 11, we show two primitive context descriptions from our sales scenario.

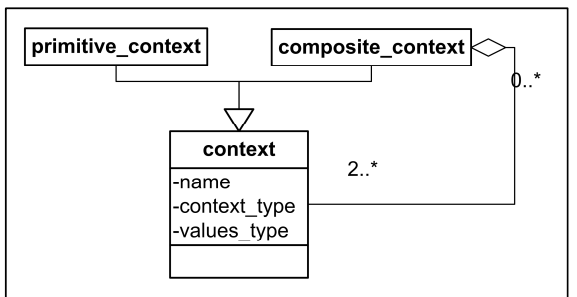

Figure 10. Context description meta-model of DSOPL-ADL

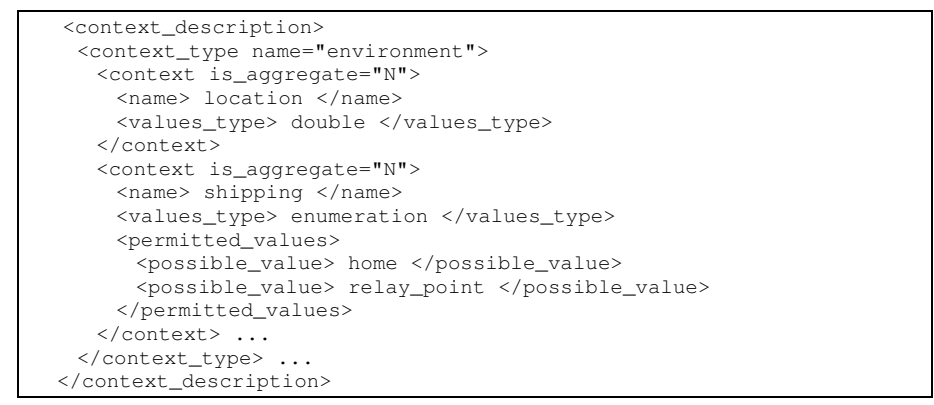

Figure 11. Some context descriptions from sales scenario

\section{F. Configuration description}

In traditional architectures, where environment is considered stable, services are selected and composed at design time. In contrast, in dynamic environment, parts of the software can be instantiated or evolved at runtime. Therefore, we need to maintain, in addition to structural information, architectural information of the running system. The configuration section of DSOPL-ADL allows describing all the configuration rules to generate valid architectures. A valid architecture is a concrete architecture whose services and connections comply with configuration rules.

The configuration description section of DSOPL-ADL has an initialization sub-section, where all static elements (services and connections) in addition to alternatives, whose variation_time="compile_time", are instantiated. The connection part has two references to two different service interfaces, the one that calls the information consumer_interface and the one that provides the information provider_interface. The configuration description also has a dynamic_configuration sub-section where architectural configurations are triggered based on runtime context conditions. In other words, a concrete architecture is selected through two consecutive execution levels: (1) static bind where core services are selected and bound then (2) late-binding where remaining services and variation points are bound.

In initialization sub-section, we first bind static services to the configuration in addition to their connections. In dynamic_configuration sub-section, we integrate selected instances of services by observing context changes that are specified in the condition part of the configuration rule. Fig. 12 illustrates the architectural configuration meta-model. Any partial_configuration has a name and an attribute called priority of type integer, which determines which configuration to choose in case more than one partial_configuration satisfies current conditions. At that time, the one with the higher priority is privileged. Each partial_configuration is composed of two parts; condition part and dynamic_action part. In the condition part, we specify conditions that are driven by context elements. In the dynamic_action part, we specify all dynamic activities that will be realized. Every action concerns an architectural element which can either be a service or a connection. Action_type defines the type of change that will apply on the selected element. Its values are limited to bind, unbind, activate or deactivate concerned elements.

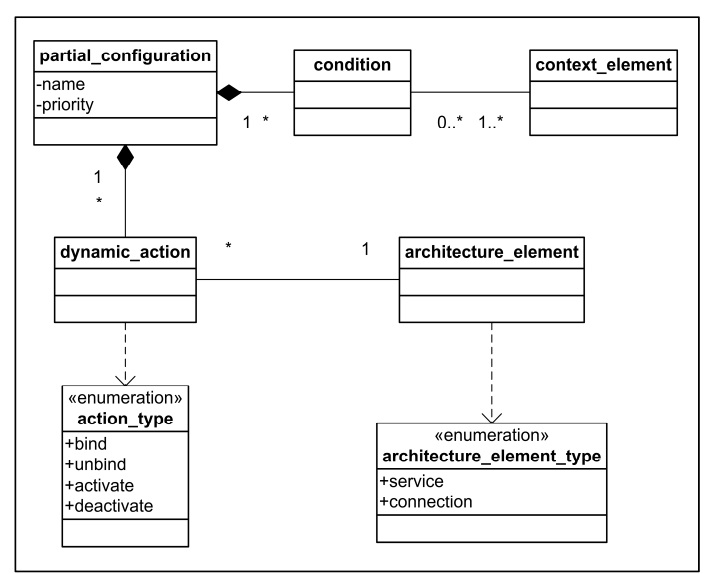

Figure 12. Configuration description meta-model of DSOPL-ADL 
In our illustrative example, customer and supply chain management services are instantiated at design time, as depicted in Fig. 13, whereas the relay point shipping service or home delivery shipping service are instantiated dynamically depending on environment's conditions.

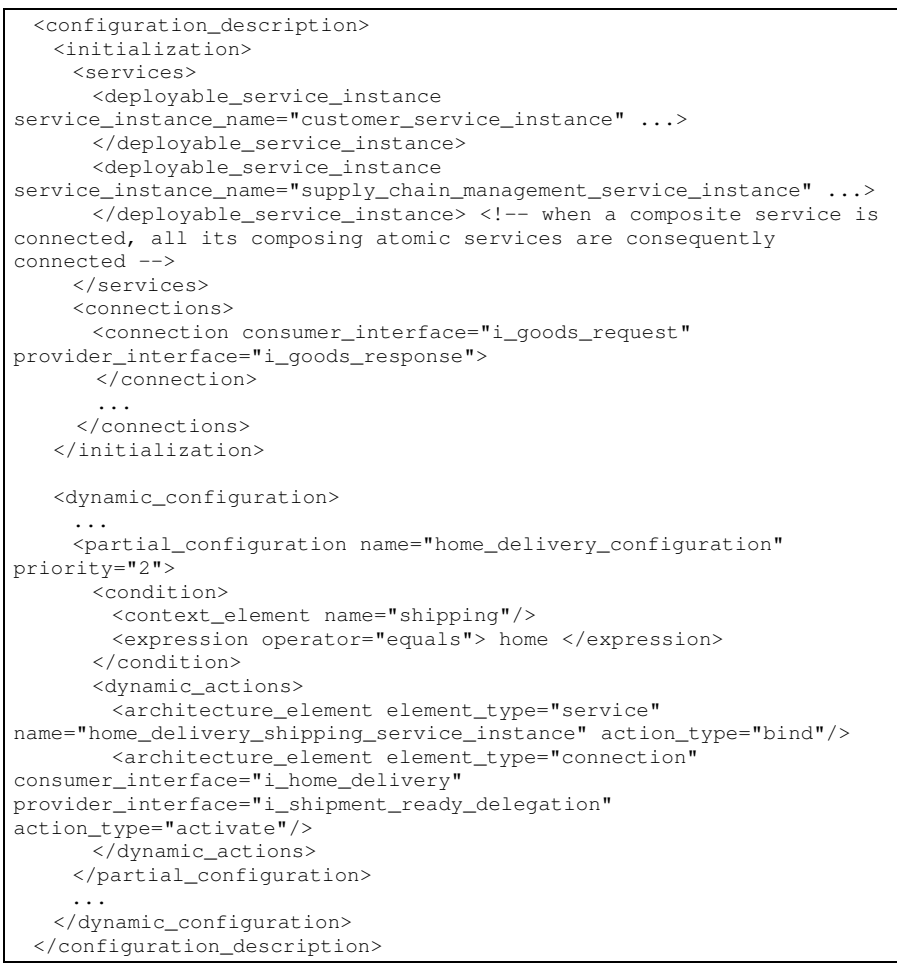

Figure 13. Configuration description of sales scenario

\section{CONCLUSION AND PERSPECTIVES}

We have presented DSOPL-ADL, an architectural language that allows the runtime variability of a service based product lines system to be modeled. To manage the runtime variability of such service based systems at architectural level, we have proposed a modular language called DSOPL-ADL which is structured and composed of four sections; structural, variability, context and configuration. For each part, its metamodel was presented and discussed in detail through an illustrative example.

It is worth noting that we have perceived variability in this work from a spatial perspective and not temporal, that is why we have only considered describing variation points and alternatives and have intentionally eliminated versioning aspect. Another point is that during late binding, we do not use any real-time configuration verification mechanisms. However, we assume that pre-conditions and post-conditions assure a valid configuration.

We are working on generating BPEL process from DSOPL architecture. As a future work; we intend to build a modeling tool for DSOPL-ADL and to conduct more experiments in order to completely evaluate our approach.

\section{REFERENCES}

[1] P. Clements, D. Garlan, F. Bachmann, J. Ivers, J. Stafford, L. Bass, P. Merson. Documenting software architectures: views and beyond, 2nd edition. Addison-Wesley Professional, 2010.

[2] B. Mohabbati, B. Asadi, D. Gašević, J. Lee. Software Product Line Engineering to Develop Variant-Rich Web Services. In Web Services Foundations, pp. 535-562. Springer New York, 2014.

[3] P. Clements, L. Northrop. Software product lines: Practices and Patterns. Addison-Wesley, 2001.

[4] M. Galster, P. Avgeriou, D. Weyns, T. Männistö. Variability in software architecture: current practice and challenges. SIGSOFT Softw. Eng. Notes, vol. 36, no. 5, pp. 30-32, September 2011.

[5] M. P. Papazoglou, W.-J. Heuvel. Service oriented architectures: approaches, technologies and research issues. The VLDB Journal, vol. 16, no. 3, pp. 389-415, 2007.

[6] N. Medvidovic. ADLs and dynamic architecture changes. In Joint proceedings of ISAW-2 \& Viewpoints '96 on SIGSOFT '96.

[7] J. Lee, G. Kotonya, D. Robinson. Engineering Service-Based Dynamic Software Product Lines. Computer, vol.45, no.10, pp. 49-55, Oct, 2012.

[8] N. Medvidovic, R.N. Taylor. A classification and comparison framework for software architecture description languages. IEEE Transactions on Software Engineering, vol.26, no.1, pp.70-93, Jan 2000.

[9] N. Medvidovic, P. Oreizy, J. E. Robbins, R.N. Taylor. Using objectoriented typing to support architectural design in the $\mathrm{C} 2$ style. In Proceedings of SIGSOFT '96, pp. 24-32, 1996.

[10] J. Magee, N. Dulay, S. Eisenbach, J. Kramer. Specifying Distributed Software Architectures. In Proceedings of the 5th European Software Engineering Conference, pp. 137-153, 1995.

[11] F. Oquendo. $\pi$-ADL: An architecture description language based on the higher-order typed $\pi$-calculus for specifying dynamic and mobile software architectures. ACM SIGSOFT, pp. 1-14, 2004.

[12] D.C. Luckham, J.J. Kenney, L.M. Augustin, J. Vera, D. Bryan, W. Mann. Specification and Analysis of System Architecture Using Rapide. IEEE Trans. Software Eng., vol. 21, no. 4, pp. 336-355, Apr. 1995.

[13] A. Joolia, T. Batista, G. Coulson, A.T.A. Gomes. Mapping ADL Specifications to an Efficient and Reconfigurable Runtime Component Platform. WICSA'05, pp.131-140, 2005.

[14] R. Allen, R. Douence, D. Garlan. Specifying dynamism in software architectures. In Proceedings of the Workshop on Foundations of Component-Based Systems, Zurich, Switzerland, pp. 11-22. 1997.

[15] C. Cetina, P. Trinidad, V. Pelechano, A. Ruiz-Corts. An Architectural Discussion on DSPL. 2nd International Workshop on Dynamic Software Product Lines (DSPL08). Limerick, Ireland, 2008.

[16] E.Y. Nakagawa. Reference architectures and variability: current status and future perspectives. In Proceedings of the WICSA/ECSA, 2012.

[17] E.M. Dashofy, A. van der Hoek, R.N. Taylor. An Infrastructure for the Rapid Development of XML-based Architecture Description Languages. In ICSE2002, Orlando, Florida, 2002.

[18] R. van Ommering, F. van der Linden, J. Kramer, J. Magee. The Koala Component Model for Consumer Electronics Software. Computer 33, 3, pp. 78-85, March 2000.

[19] F. Oquendo. $\pi$-ADL for WS-Composition: A Service-Oriented Architecture Description Language for the Formal Development of Dynamic Web Service Compositions. In: SBCARS, pp. 52-66, 2008.

[20] X. Jia, S. Ying, H. Cao, D. Xie. A New Architecture Description Language for Service-Oriented Architecture. In Sixth International Conf. on Grid and Cooperative Computing GCC, pp. 96-103, 2007.

[21] M. Galster. Describing variability in service-oriented software product lines. In Proceedings of the ECSA'10, pp. 344-350, 2010.

[22] R. Capilla, et al. An overview of Dynamic Software Product Line architectures and techniques: Observations from research and industry. Journal of Systems and Software, vol. 91, pp. 3-23, May 2014. 